\title{
What Uses of Connected Mobile Microsensors of Air Pollution in the General and Vulnerable Populations?
}

\author{
D Charpin ${ }^{1 *}$ and R Collomp ${ }^{2}$ \\ ${ }^{1}$ French Clean Air Association and Aix-Marseille University, France \\ ${ }^{2}$ French Clean Air Association and Nice Hospitals and University, France
}

*Corresponding author: Denis Charpin, Association pour la Prévention de la Pollution Atmosphérique, Hotel Technologique, 45 rue Joliot-Curie, 13013 Marseille, France.

To Cite This Article: D Charpin, R Collomp, What Uses of Connected Mobile Microsensors of Air Pollution in the General and Vulnerable Populations?. Am J Biomed Sci \& Res. 2021 - 11(4). AJBSR.MS.ID.001650. DOI: 10.34297/AJBSR.2021.11.001650.

Received: 阱 December 21, 2020; Published: 些 January 12, 2021

\begin{abstract}
This review article summarizes the potential uses of mobile microsensors by the public and some vulnerable populations. Firstly, use of such sensors could help promote individual behaviors aimed at limiting exposures and the generation of air pollutants. However, access to the best information, if taken alone, does not necessarily translate into a change in individual behavior. Knowledge can promote changes in behavior when there are measures available that can be implemented and if the benefits of the actions can be verified in the short run and if they are combined with coaching by scientific or medical experts. Secondly, the use of mobile sensors could promote collective behavior and influence public policies that promote air quality. Nowadays, information intended for the public is only provided through media outlets during periods of elevated air pollution and consists of restrictive advice that are not specifically relevant for at-risk groups. Mobile microsensor users could establish forums and share information regarding air quality. Thirdly, the use of mobile microsensors is likely to provide more accurate estimations of exposure in epidemiological studies and increase the density of the air quality monitoring network.
\end{abstract}

Keywords: Air Pollution, Citizen Science, Microsensors

\section{Background}

Outdoor and indoor air pollution are responsible for extensive morbidity and premature mortality, hence justifying the name assigned by the WHO of "the invisible killer" [1]. These forms of pollution nowadays represent the second-highest concern people have regarding environmental issues, after global warming [2]. There are presently real societal needs in terms of knowledge of exposures and their health impacts [3].

The area for research of "Air pollution and health" remains, even nowadays, complex for several reasons: there is not a single pollutant, but a large number of pollutants. These interact with each other, leading to a mixture of both primary and secondary pollutants. The exposure is heterogeneous over time linked with different activities and locations (requiring continuous measurements) and in space (requiring measurements with an as fine as possible geographical network) but also in terms of sensitivity for a given individual (thus highlighting the need for individual data). There are different regulatory threshold levels (national, European, global) for certain pollutants but, in terms of health, there is no "safe" level. The diseases likely to be linked with atmospheric pollution are multifactorial, with the environment being involved in a partial and/or indirect manner in their occurrence.

In this review, we do not consider fixed sensors that are smaller and less expensive relative to traditional measurement devices, also called "microsensors", which have presently broadened the coverage of monitoring by networks that monitor air quality. Those sensors are used in "targeted mobile monitoring", which is a coordinated, goal-driven approach in which measurements are performed with a specific goal in mind, whereas "opportunistic mobile monitoring" [4] uses existing mobile monitoring in people's daily routines.

This review follows the "State of the Art" approach, which addresses grey literature (from Google search engine) in addition to 
the scientific literature. Keywords used in PubMed up to December 2020 were as follows: low-cost sensors/portable sensors/citizen science and air quality.

The relevance of connected mobile microsensors entails three synergistic objectives in regard to air quality.

\section{Promoting Individual Behavior Aimed at Limiting Exposure and the Generation of Atmospheric Pollutants}

\section{Being Less Exposed to Air Pollution}

Most citizens do not currently have ready access to data regarding the levels of pollutants. There is a significant gap between how agencies report data and the people who would benefit from access to that knowledge. Moreover, indoor air pollution should be taken into account since, on average, $90 \%$ of contemporary life is spent indoors [5]. Individuals can consult the websites of the public agencies in charge of monitoring air quality that provide online access to: maps of the area colored according to the estimated concentrations, generally an index of the exposure according to the national regulations in effect, and sometimes precise measurements derived from their respective measurement stations. In addition to measurements of regulated pollutants, in some cases, the measurements also comprise the levels of other pollutants. Thus, with connected microsensors, the indicated data can be in regard to daily or hourly measurements, while it can also be extrapolated by means of statistical projections and forecasts for subsequent days. Another modality of access to data is by means of smartphone applications.

The creators of these applications have generally put a lot of effort into optimizing their ergonomic properties so as to promote their use and especially exploitation of the data presented and any associated messages: Thus, particular attention has been paid to the overall graphics and colors; simplicity of the screens; intuitiveness of the functions offered; ease of navigation between screens; geographical presentation of the maps, which are sometimes accompanied by photographs to facilitate location; the display, not only of the values for the various pollutants, as the personal thresholds also need to be shown, as do the general pollution levels (for example good quality, average quality and poor quality), by means of various readily understandable symbols (e.g. smiley face emojis); the presentation of prior readings in the form of graphs to monitor changes; whenever possible, the display of alerts when thresholds are exceeded; and clarity and simplicity of the messages and the advice that is provided.

Most often, GPS positioning integrated with a smartphone allows the data corresponding to one's position to be obtained automatically. Other services can be provided by these applications such as recommended routes and associated advice. However, in parallel with this ease of access, there are several issues, which most users are not always aware of. Where do the data sources come from so as to ensure their metrological quality, how is the collected data processed if it is not strictly government regulatory network data? Is the associated advice sound? Is there an associated business model geared to a device being purchased (for example air purifier)?

The use of mobile sensors, and their associated applications is, to a certain extent, a response to these limitations.

If mobile sensors are indeed subject to exactly the same issues of data transparency and commercial interests, the possible problem of the quality of data sources, if non-governmental, is removed. Indeed, they allow the levels of outdoor and indoor air pollution to be known in a factual and reliable manner at the individual level and in real time, as they are obtained directly from the device evaluated upstream of sufficient metrological quality. Thus, the Ambassad'Air testing [6] carried out annually at Rennes (France), among voluntary residents, shows the impact of activities such as cycling in a traffic intersection (Figure 1), cooking food (Figure 2), or smoking of a cigarette in terms of exposure to fine dust. Another study [7], based on monitoring of a group of 14 adult volunteers, concluded that the time spent at home engaged in domestic chores, particularly the cooking of food, is associated with substantial exposure to PM2.5. In this study, the characteristics of the dwelling did not influence the level of exposure. The proximity of the dwelling to thoroughfares had an impact on the level of PM2.5. Other studies have emphasized the relevance of airing out one's home, particularly when engaged in cooking (barbequing) [8], of staying indoors in case of peaks of pollution [9], of better use of one's stove [10], and of adequate ventilation when using the fireplace and avoiding that children then stay indoors [11]. The Citisense program [12] is interesting because its objective is to learn how sensors can be integrated into the daily activity of different citizens and how participants perceive their environment through access to real-time air quality readings. The authors have reported several impacts of this program on how air pollution data is processed and used. First of all, the data from the sensors made it possible to confirm factually known exposure levels as risk areas, as well as to become aware of the existence of pollution in supposedly "healthy" areas, thus corresponding to the acquisition of an ability to verify pollution expectations. Additionally, the knowledge of pollution levels via the sensors challenged the previously held beliefs about air and pollution distribution. Initial conceptual models, such as the notion that the distribution of air pollution is uniform or that air pollution levels do not change over short distances, proved to be inconsistent with the data collected by the sensor. Faced with high levels of pollution, the participants did not remain passive, but instead searched for the origins of the 
pollution, and they developed a better "intuitive" sense of the actual source of pollution. Kim and Paulus [8] discussed how the ability to identify the source of high pollution readings is a key factor in designing systems that enable change and to avoid triggering a sense of being powerless. Finally, as reported by the Citisense authors, the participants stopped checking their phones at regular intervals as the experiment progressed, and instead they did so only when they were prompted by an anomalous observation, such as walking past a new construction site or driving behind a vehicle emitting copious amounts of exhaust fumes. In summary, according to Bales et al. [12], the observations collected during the study are evidence of the intellectual effort that participants made to process the readings they observed when they did not originally have any particular skills in this regard. In addition to relating sensor readings to the environmental context, the participants were able to use the sensors to understand their own physical responses to their environment as a potential individual source of pollution. Ultimately, the authors did not observe a sense of being powerless in regard to the measured pollution, but rather an awareness and empowerment of the participants. Indeed, the fact that use of the sensors made poor air quality "visible" and quantifiable helped citizens feel sufficiently informed to make informed personal choices as well as to become more involved in improvement of their communities. However, a study by Oltra et al. [13] is at variance with this conclusion: they included 12 participants who used realtime NO2 sensors over a period of 7 days and compared them to 16 participants who were only given documentary informations about air pollution. In both groups, beliefs of controllability and self-efficacy were equally low.

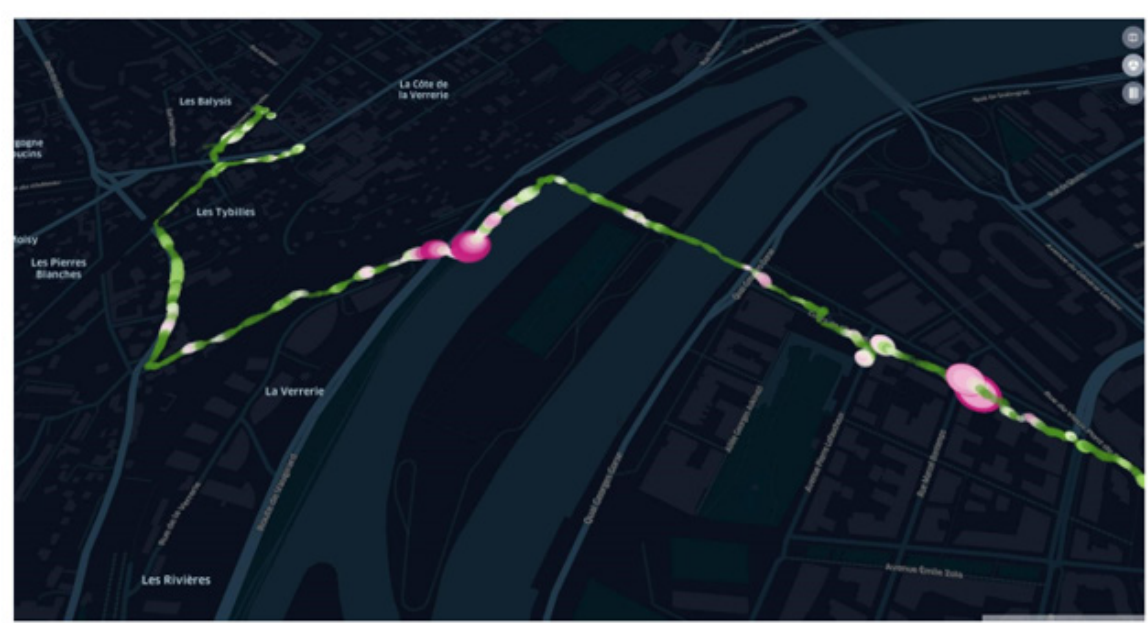

Figure 1: The cyclist follows a route that has little pollution (green line). The line widens and becomes pink when he (she) is at an intersection with heavy vehicular traffic [6].

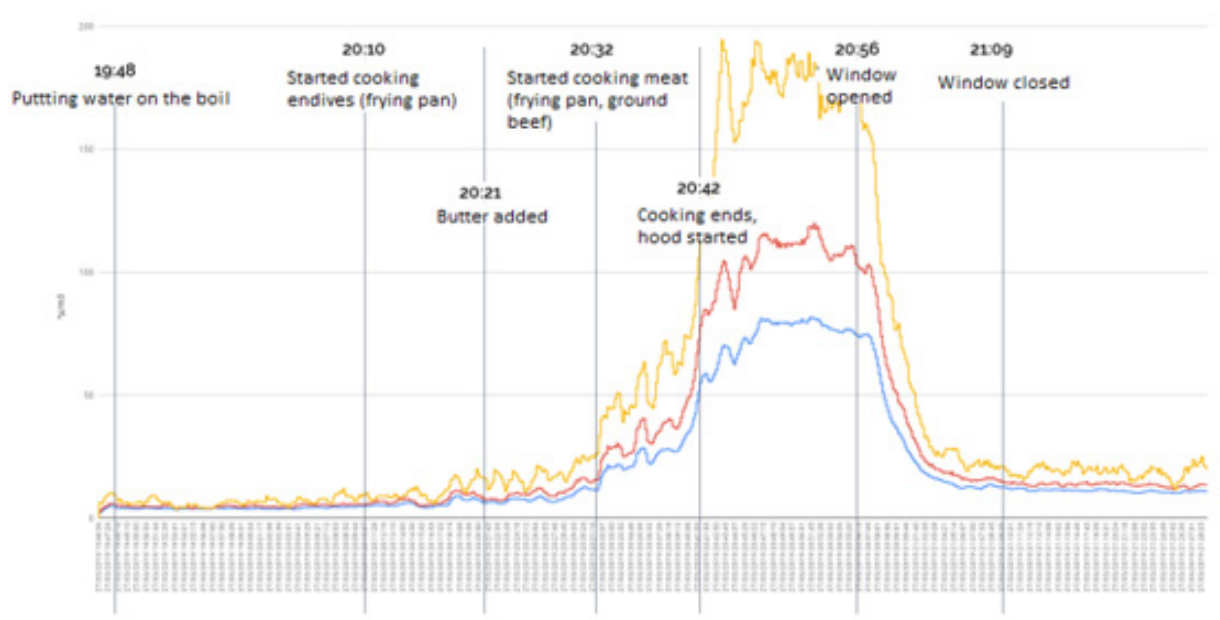

Figure 2: Change in the airborne concentration of fine particulate matter (PM2.5) in the kitchen during culinary activities. The inscriptions at the bottom of the figure correspond to the chronological sequence of the observation, with measurements carried out every minute (personal observation). The 3 curves refer to PM10 (upper curve), PM2.5 (middle curve) and PM1.0 (lower curve) measurements. 


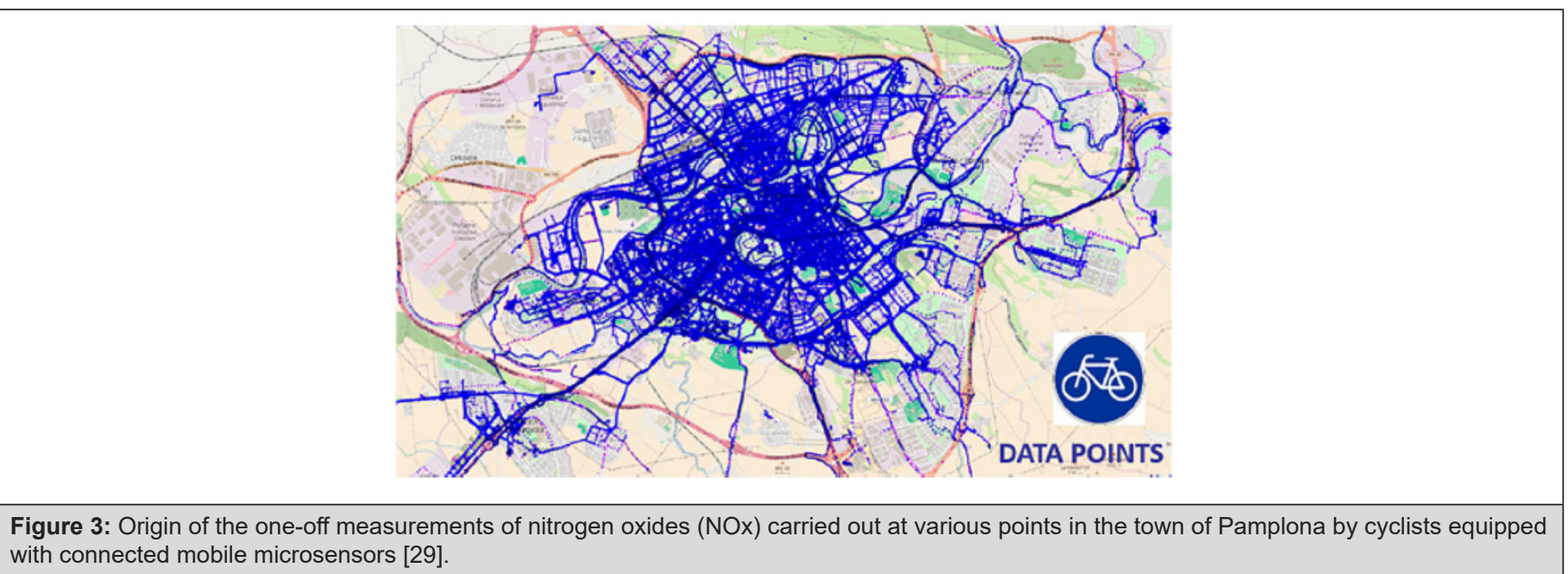

Aside from these "ad hoc" studies, several applications nowadays allow a smartphone to be used to access information regarding the levels of pollution in one's immediate environment and to choose a walking route or a site for engaging in sporting activities that is less exposed, although this is beyond the scope of the question addressed here since these data regarding pollution are not measured by the device itself but transmitted by the application based on sources of data that have been more or less identified.

This objective of limiting exposure to atmospheric pollutants is naturally beneficial for the entire population, but it is even more relevant for groups that are particularly vulnerable in regard to air quality: patients suffering from a respiratory pathology (asthma, chronic obstructive pulmonary disease, etc.) or cardiovascular disease, pregnant women, infants and young children, and the elderly. These populations are likely to be the main users of such connected mobile microsensors. For these vulnerable populations, the potential interest of the sensors would be to combine the measurements taken by the system with adapted and personalized health advice in order to facilitate the targeted behavior change. This can be done automatically according to simple algorithms developed upstream, combining the pollution level and personal profile entered in the smartphone application. They can also be provided individually, based on the same data or enriched by declarations of clinical signs via the smartphone application, for example via remote monitoring and teleconsultation platforms, following the model of other diseases using connected devices such as diabetes, for example. In these particular situations, since the data from the sensors lead to medical decisions, their qualities, directly linked to the metrological quality of the sensors, will have to be particularly verified upstream.

\section{Polluting Less}

That is to say, more readily adopting individual behaviors that enhance air quality, in terms of the environment or health, as a result of being cognizant of the level of pollution generated by one's activities. Thus, avoiding heating with a wood stove [14], choosing an appropriate fuel for cooking, avoiding the use of incense sticks or scented candles [15], and adopting behaviors such as ridesharing or the use of public transport $[9,16]$.

\section{Being Less Exposed, Polluting Less: Does the Best Information Automatically Translate into a Change in Individual Behavior?}

Modification of individual behaviors is a complex issue no matter what is being targeted: mobility, stopping smoking, etc. To have a long-term impact on a behavior, it is not enough to "know", it needs to involve "putting it into practice". It relies on several dimensions: cognitive (knowledge, learning, skills, concepts), emotional, metacognitive (one's own thought process), and infracognitive (usual reflexive thoughts). A specific difficulty with changing behavior in regard to air quality is the clash between the requirements: changing one's behavior here and now, by means of constraints, for a health benefit expected in the medium to long term (and not immediate, except in vulnerable populations). Moreover, unlike other issues such as nutrition and the objective of "healthy eating", for which there is the option to choose what we eat and our consumption, there is a certain fatality in regard to air quality. "What can I do about it as I have no choice but to breathe the air around me". The concept of being in a position, whenever this is possible, such as with physical activities, to choose the best place and the right time to engage in these activities, is currently largely unknown. Thus, even though, as described above, this type of pollution is now a high-priority concern for citizens, the actual observed changes in behavior have remained minimal to date.

The report of the French Agency for the Environment and Energy Management (ADEME) [17], based on 80 experiments and 20 publications, compiled a certain number of negative findings in the literature: only $15 \%$ of the people cognizant of their exposure to a peak of pollution implemented preventative actions [11], the 
participants most aware of the deleterious effects of pollutants nonetheless still have a passive attitude [18], mothers who have been made aware of an air quality issue by an information campaign do not, however, reduce the sources of domestic pollution [14]. In summary, this report estimated that the knowledge of the level of pollution and of associated health risks is not sufficient to induce a lasting change in behavior. A sensor alone is not enough to implement actions. The ease of adopting practices and the sharing of information through Facebook or Twitter were key points in the dynamics of changes in behavior. In CitiSense, in addition to online sharing, the participants also frequently shared information with people around them [12]. Digital technologies benefit from strong popular support in order to make subjects aware of air quality, and for developing learning regarding the capture and/or the interpretation of the measurements. The results differ according to the two main categories of individuals participating in the experimentations: individuals already aware of air quality, versus volunteers or uninformed individuals who are asked to participate.

Taking action after becoming aware of the levels of pollution provided by connected microsensors requires that three conditions are met:

a) first of all, being cognizant of the sources of pollution [8]

b) secondly, having measures available that can readily be implemented from a technical, economic, and psychological perspective. For example, an action that adversely affects sources of revenue or that disrupts set habits $[8,10]$ will not be implemented often

c) lastly, having the means to, in the short-run, verify the efficacy of actions in terms of individual benefits

Furthermore, the involvement of volunteers in a network or platform for sharing of data is a gage of continued motivation.

\section{Promoting a Collective Behavior That is Beneficial in terms of Air Quality}

The availability of information for the general population is nowadays essentially limited to periods of peak pollution, while the greatest health risk is linked with chronic exposure. As it is nearly always disseminated in an isolated manner, with associated recommendations, it ends up triggering anxiety [19], and the information can hence be counterproductive [20]. During periods of poor air quality, the transmitted information is often associated with the implementation of constraints, particularly in terms of mobility: restrictions on driving and traffic. These measures are often not well received by the general public, as they are acutely aware of the immediate impacts, but they are less cognizant of the broader environmental or health benefits. There could be a large discrepancy between the perceived air quality and objective measurements, leading the public not to support, or even oppose, traffic-reduction measures. This occurred in the southwest part of Potsdam, the capital city of the federal state of Brandenburg, Germany where a study was conducted during the trial implementation of traffic measures aimed at reducing individual vehicular traffic and encouraging the modal share of alternative forms of urban mobility, especially by walking, cycling and public transport [3]. Moreover, the dissemination of information is very extensive: mostly by the radio, as well as by roadside signs. There are no messages or dissemination means to ensure that the most vulnerable individuals have properly received the messages, and more importantly, that they have understood them. In summary, the information is not really targeted or tailored and hence not evaluable/evaluated, and consequently the impact of their cost is not known.

The use of individual mobile sensors, thanks to personalized information on the likelihood of exposure and in real-time, could in part address these limitations, as generating increased awareness in the general population of the importance of maintaining good indoor and outdoor air quality would presumably lead to better acceptance of the imposed constraints, such the restriction of traffic in certain areas and on certain days, that the public authorities increasingly use in relation to promoting health in keeping with the national and European requirements.

Furthermore, the report of the ADEME indicates that the participants in the Ambassad'Air program [6] raised the level of awareness of those they are in close contact with, their work colleagues, and even strangers, by sharing their measurements on social networks. This educational function stems from forums based on sharing of the information, such as Mobicit'Air, "Open Sense II", "Air Casting", and "Air Quality Egg" (Table 1).

Table 1: List of the participatory projects involving measurement of pollutants using mobile microsensors [17].

\begin{tabular}{|c|c|c|c|c|}
\hline Name & Geographical Area & Type of Entity & Outdoor/ Indoor air & Pollutants Measured \\
\hline City Sense & European cities (Barcelona, Ostrava) & Research Institute & Both & $\mathrm{NOx}, \mathrm{Ozone}$ \\
\hline Air Casting & USA, Worldwide & $\mathrm{NGO}$ & Both & $\mathrm{PM}_{2.5}, \mathrm{CO}, \mathrm{NO}_{2}$ \\
\hline OPAL Air Survey & United Kingdom & Research Institute & Outdoor air & $\mathrm{NOx}, \mathrm{NH}_{3}$ \\
\hline Air Quality Egg & Worldwide & Collaborative Group & Both & $\mathrm{NO}_{2}, \mathrm{CO}$ \\
\hline Open Sense II & Worldwide & Research Institute & Outdoor air & $\mathrm{O}_{3}, \mathrm{NO}_{2}, \mathrm{CO}_{,} \mathrm{CO}_{2}, \mathrm{PM}_{2.5}, \mathrm{UFP}$ \\
\hline Mobicit'Air & Grenoble city (France) & Public air quality network & Mostly Outdoor air & $\mathrm{PM}_{2.5}, \mathrm{CO}_{2} \mathrm{NO}_{2}$ \\
\hline
\end{tabular}




\begin{tabular}{|c|c|c|c|c|}
\hline iSPEX & Netherlands & University & Both & Particles \\
\hline Ambassad'Air & Rennes city (France) & Townhall & Outdoor air & PM $_{10^{\prime}}$ PM $_{2.5}$ \\
\hline Citizen Sense & Pennsylvenia (USA) & University, Public Agencies & Both & BTEX, PM $_{2.5}$ \\
\hline
\end{tabular}

Influencing Public Policies and Environmental Decision Making

Aside from changes in individual behavior, grassroots actions have been able to be implemented, such as the signing of petitions or participation in campaigns for environmental justice. The Gabrys et al. paper [21] addressed the question of how readily accessible lowcost technology can be used to create a new type of environmental data, how to exploit them, and how the latter can generate new practices beyond the traditional uses of environmental data. The ultimate aim is to promote the implementation of collaborative projects at a regional level that includes all of the stakeholders involved: communities, individuals, industry partners, and health professionals. It also focuses on how the citizen data can be interpreted and how they could bring about political change. Hubbell et al. [22] called to mind several reports which emphasized the potential of citizen-based air monitoring to impact broader policy actions and change industrial processes. However, these reports also give warning about a possible transfer responsibility for risk reduction from the polluters and public authorities to the vulnerable populations themselves, potentially exacerbating reduction of empowerment because of administrative hurdles.

\section{Improving Knowledge Regarding Air Quality}

\section{Providing a More Accurate Estimation of the Exposure in Epidemiological Studies}

The sampling currently used by governments employs stationary air monitoring systems to estimate regional air quality. This is an important starting point but conveys little details about actual individual-level exposure. Regional air quality assessment is conducted by widely dispersed sensors, with many large cities covered by only a handful of stations. Air quality can be highly variable across neighboring sites due to differences in the regional geography, industrial areas, the weather, and traffic patterns. Furthermore, for pragmatic reasons, regional air quality monitors are often placed in locations that are far from where people actually spend most of their time, such as on top of buildings, away from major roads and freeways, and -needless to say- not in people's homes. As a result, current monitoring methods are limited in their ability to inform individuals of the elevated exposures that they may encounter in their daily lives while stuck in traffic, sleeping in their homes, or walking along a busy road [12] Air pollutants have, by their very nature, a high degree of spatio-temporal variability. Questionnaires used in epidemiological studies have limitations in terms of assessing exposures through diaries and to evaluate activity patterns. The various activities that people engage in during the day lead to overall exposures that cannot be adequately determined when only home address-based concentrations are estimated [23]. Mobile microsensors could provide opportunities to understand exposures that vary in time and space and to measure activity patterns. The exposure data attributed to an individual based on measurements taken at set sites or based on models of dispersion can hence provide inaccurate values, which decreases the power of an epidemiological study. Mobile microsensors could decrease the measurement errors in epidemiological studies and provide valid data for citizen science studies [24,25]. For example, in one study [23], the average exposure while traveling accounted for $6 \%$ of the time whereas it accounted for $24 \%$ of the daily NO2 exposure. Vedal et al. [26] put forward a design of air pollution monitoring in Beijing for a cohort health study. They considered that a campaign that includes a mobile monitoring component, when coupled with currently available spatio-temporal methods, should be strongly considered, as each of these has relative advantages and disadvantages. In multicenter studies, region-specific calibration should be performed when meteorological and environmental conditions differ [27]. This paper highlights the need for performing calibration based on regional scale comparisons to date references. and it cautions against using manufacturer-provided general calibration factors. Finally, while air pollution data from sensors can be cross-referenced with clinical data, especially those reported by sensor users, their joint exploitation allows further development of detailed knowledge of the impact of air pollution on the health of the general population or vulnerable populations.

\section{Increasing the Density of the Network for Monitoring Air Quality}

The high costs of installing and maintaining reference monitoring stations explain that, even in developed countries, few areas of large cities are covered, while in small towns and rural areas there may be no such stations [28]. In the introduction, we also indicated that the official agencies for monitoring of air quality are presently increasing the density of the networks by relying on less expensive fixed sensors. Moreover, mobile microsensors provide new opportunities to obtain information regarding air pollution at high spatial resolution in real-time. Furthermore, there have been trials that consisted of equipping volunteers recruited in different parts of a town. Such a trial has been carried out in the town of Pamplona in Spain. This has allowed a very thorough picture to be obtained of the levels of nitrogen oxides in the town [29] (Figure 3). The Mobicit'Air trial [30], carried out by the air quality monitoring network of the Auvergne Rhône-Alpes region in Grenoble, has concluded that "these technologies, as a result of the large number 
of measurements carried out, supply and improve the modeling of air quality, provided that the metrological quality is adequate". According to the EPA in the USA, "more, but less precise, air quality measurements can supplement the measurement data from established monitoring stations" [31]. In developing countries, due to the lack of air pollution data, such low-cost sensors represent an opportunity to fill this gap. However, there is still a need to obtain standard reference equipment such as calibration instruments [32].

\section{Conclusion and Perspectives}

The "ideal" connected mobile microsensor should have the following qualities: good metrological properties (accuracy and precision), ease of use (simplicity, ergonomics, lightweight, rugged, low energy consumption, clear presentation of the data, compatibility of the software/smartphone), and economical (low cost). None of the currently available devices meets all of these criteria. However, their standards are regularly and rapidly being improved. Independent exterior analyses are needed and are being implemented. However, there are still no "norms" or single and validated evaluation grids.

Furthermore, other than the intrinsic quality of the sensors, an effort needs to be made to ensure that the users are sufficiently trained beforehand and then supervised regarding the use of connected mobile microsensors so as to minimize the risks of misinterpretation of the data, which could, in fact, lead to counterproductive results. This should take place from the start, so as to avoid the understandable but sometimes excessive anxiety in regard to the measurements collected. As explained above, the ultimate aim remains achieving a lasting change in behavior. This support could be ensured by government entities and particularly agencies for the general public, and by the health professionals (doctors, pharmacists in particular) for vulnerable populations. These professionals will also have to be trained beforehand by training programs that are under development.

Lastly, in an area that now has a high potential economic impact, another set of issues stemming from the development of the use of connected mobile microsensors pertains their use and the legal aspects in regard to the data collected and disseminated in terms of ownership and management of personal data, particularly as these will be rapidly coupled, as indicated, to health data. Thus, mobile microsensors technology is creating new challenges for information security and privacy [33].

\section{References}

1. Cohen AJ, Brauer M, Burnett R, Anderson HR, Frostad J, et al. (2015) Estimates and 25-year trends of the global burden of disease attributable to ambient air pollution: an analysis of data from the Global Burden of Diseases Study. Lancet 389(10082): 1907-1918.
2. (2019) French National Institute of Statistics and Economical Studies.

3. Schmitz S, Weiand L, Becker S, Niehoff N, Schartzbach F, et al. (2018) An assessment of perceptions of air quality surrounding the implementation of a traffic-reduction measure in a local urban environment. Sustained Cities Society 41: 525-537.

4. Van den Bossche J, Theunis J, Elen B, Peters J, Botteldooren D, et al. (2016) Opportunistic mobile air pollution monitoring: A case study with city wardens in Antwerp. Atmospheric Environment 141: 408-421.

5. Jiang Y, Li K, Tian L, Piedrahita R, Yun X, et al. (2011) MASQ: a personalized mobile sensing system for indoor air quality monitoring. Proceedings of the 13th international conference on ubiquitous computing pp.271-280.

6. Calvez M (2018) What is citizen expertise in environmental health ?. Questions about the Rennes Ambassad'Air experiment. Environment Health Risks 17(5): 498-504.

7. Mazehari M, Clifford S, Jayaratne R, Mokhtar M, Fuoco FBG, et al. (2014) School children's personal exposure to ultrafine particles in the urban environment. Environ Sci Technol 48(1): 113-120.

8. Kim S, Paulus E (2009) In Air: Measuring and visualizing indoor air quality. In: Proceed of the SIGCHI Conference on Ubiquitous Human Factors in Computing Systems.

9. Jin W (2006) A smart indoor air quality sensor network. Proceed SPIE 6174 Smart Structures and Materials 617440: 1861-1870.

10. Barnes BR (2014) Behavorial change, indoor air pollution and child respiratory health in developing countries: A Review. Int J Environ Res Public Health 11(5): 4607-4618.

11. Semenza JC, Wilson DJ, Parra J, Bontempo BD, Hart M, et al. (2008) Public perception and behavior change in relationship to hot weather and air pollution. Environ Res 107(3): 401-411.

12. Bales E, Nikzad N, Ziftci C, Quick N, Griswold W, et al. (2014) Personal pollution monitoring: Mobile, real-time, air-quality in daily life. ACM DL DIGILAL LIBRARY 23(2): 1-10.

13. Oltra C, Sala R, Boso A, Lopez Asensio S (2017) Public engagement on urban air pollution: an exploratory study of two interventions. Environ Monit Assess 189(6): 296.

14. Tun KM, Win H, Ohnmar T, Zaw AKNT, Nyat KKS, et al. (2005) Indoor air pollution: Impact on interventions on acute respiratory infection (ARI) in children. Regional Health Forum 9(1): 30-36.

15. Wakefield SE, Elliot SJ, Cole DC, Eyles JD (2001) Environmental risk and (re)action: Air quality, health and civic involvement in an industrial neighborhood. Health Place 7(3): 163-177.

16. Mei NS, Choong WN, Ahamad R (2016) Environmental awareness and behaviour index for Malaysia. Procedia-Social Behavior Sciences 222: 668-675.

17. ADEME (2017) Relationships between personal air pollution data, change in behavior, and implementation of practices beneficial to air quality.

18. Saïdi N, Planchon M, Allard L (2017) Study of the links between individual data, behavior change and the implementation of practices favorable to air quality. Rapport pp.1-102.

19. Zeidner M, Shechter M (1988) Psychological responses to air pollution: Some personality and demographic correlates. J Environ Psychol 8 (3): 191-208.

20. Lyons RA, Rodgers SE, Thomas S, Bailey R, Brunt H, et al. (2016) Effect of air pollution personal alert system on health service usage in high risk: a quasi-experimental study using linked data. J Epidemiol Community Health 70(12): 1184-1190. 
21. Gabrys J, Pritchard H, Barratt B (2016) Just good enough data: Figuring data citizens through air pollution sensing and data stories. Big Data Society pp.1-14.

22. Hubbell BJ, Kaufman A, Rivers L, Schulte K, Hagler G, et al. (2018) Understanding social and behavioral drivers and impacts of air quality sensor use. Sci Total Environ 621: 886-894.

23. de Nazelle A, Seto E, Donaire Gonzalez D, Mendez M, Matamala J, et al. (2013) Improving estimates of air pollution exposure through ubiquitous sensing technologies. Environ Pollut 176(5): 92-99.

24. Zeger SL, Thomas D, Dominici F, Samet JM, Schwartz J, et al. (2020) Exposure measurement error in time-series studies of air pollution: concepts and consequences. Environ Health Perspect 108(5): 419-426.

25. Jerrett M, Donaire Gonzales D, Popoola O, Jones R, Cohen RC, et al. (2017) Validating novel air pollution sensors to improve estimates for epidemiological analyses and citizen science. Environ Research 158(10): 286-294.

26. Vedal S, Han B, Xu J, Szpiro A, Bai Z (2017) Design of an air pollution monitoring campaign in Beijing for application to cohort health studies. Int J Environ Res Public Health 14(12): 1580.

27. Zusman M, Schumacher CS, Gasset AJ, Spalt EW, Austin E, et al. (2020) Calibration of low-cost particulate matter sensors: Model development for a multi-city epidemiological study. Environ Int 134: 105329.
28. Castell N, Dauge FR, Schneider P, Vogt M, Lerner U, et al. (2017) Can commercially low-cost sensor platforms contribute to air quality monitoring and exposure estimates. Environ Int 99(2): 293-302.

29. Rivas E, Santiago JL, Lechon Y, Martin F, Arino A, et al. (2019) CFD modelling of air quality in Pamplona City (Spain): Assessment, spatial representativeness and health impacts valuation. Sci Total Environ 649(2): 1362-80.

30. Mobicit'Air. http://www.atmo auvergne-rhone-alpes, accessed on December 3rd, 2020.

31. Roadmap for next generation air monitoring. http://www.epa.gov, accessed December 3rd, 2020.

32. Kofi Amegah A (2018) Proliferation of low-cost sensors. What prospects for air pollution epidemiological research in Sub-Saharian Africa? Environ Pollut 241(10): 1132-1137.

33. Kapadia A, Kotz D, Triandopoulos N (2009) Opportunistic sensing: Security challenge for the new paradigm. In: Proceedings of the international conference on COMmunication systems and NETworks (COMNETS). 